\title{
Article
}

\section{Metabotropic action of postsynaptic kainate receptors triggers hippocampal LTP}

Petrovic, Milos, Viana da Silva, Silvia, Clement, James P., Vyklicky, Ladislav, Mulle, Christophe, Gonzalez-Gonzalez, Inmaculada M. and Henley, Jeremy $M$.

Available at http://clok.uclan.ac.uk/16925/

Petrovic, Milos, Viana da Silva, Silvia, Clement, James P., Vyklicky, Ladislav, Mulle, Christophe, Gonzalez-Gonzalez, Inmaculada M. and Henley, Jeremy M. (2017) Metabotropic action of postsynaptic kainate receptors triggers hippocampal LTP. Nature Neuroscience, 20 . pp. 529-539. ISSN 1097-6256

It is advisable to refer to the publisher's version if you intend to cite from the work. http://dx.doi.org/10.1038/nn.4505

For more information about UCLan's research in this area go to http://www.uclan.ac.uk/researchgroups/ and search for <name of research Group>.

For information about Research generally at UCLan please go to http://www.uclan.ac.uk/research/

All outputs in CLoK are protected by Intellectual Property Rights law, including Copyright law. Copyright, IPR and Moral Rights for the works on this site are retained by the individual authors and/or other copyright owners. Terms and conditions for use of this material are defined in the policies page.

\section{CLoK}

Central Lancashire online Knowledge www.clok.uclan.ac.uk

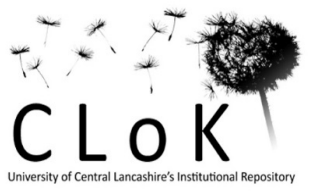




\section{$1 \quad$ Metabotropic action of postsynaptic kainate receptors triggers}

2

3

4

5

6

7

8

9

10

11

12

13

14

15

16

17

18

19

20

21

22

23

24

25

26

27

28

29

\section{hippocampal LTP}

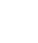

4

(1)

\author{
Milos M. Petrovic ${ }^{1,3,4,5^{*}}$, Silvia Viana da Silva ${ }^{2}$, James P. Clement ${ }^{6}$, Ladislav Vyklicky ${ }^{4}$, \\ Christophe Mulle ${ }^{2}$, Inmaculada M González-González ${ }^{11^{\star}}$, and Jeremy M. Henley ${ }^{1 \dagger^{\star}}$
}

${ }^{1}$ School of Biochemistry, University of Bristol, Bristol, UK

${ }^{2}$ Interdisciplinary Institute for Neuroscience, University of Bordeaux, Bordeaux, France.

${ }^{3}$ School of Pharmacy and Biomedical Sciences, University of Central Lancashire, Preston, UK (present address)

${ }^{4}$ Institute of Physiology, Academy of Sciences, Prague, Czech Republic

${ }^{5}$ Insitute of Medical Physiology, School of Medicine, University of Belgrade, Serbia

${ }^{6}$ Neuroscience Unit, Jawaharlal Nehru centre for Advanced Scientific Research, Bangalore, India

${ }^{*}$ Co-corresponding authors

${ }^{\dagger}$ Joint last authors

Please address correspondence to: JMH (J.M.Henley@bristol.ac.uk), IMG-G (ggonzalezmi@gmail.com) or MMP (mpetrovic@uclan.ac.uk) 


\section{Abstract}

Long-term potentiation (LTP) in the rat hippocampus is the most extensively studied cellular model for learning and memory. Induction of classical LTP involves an NMDA receptor- and calcium-dependent increase in functional synaptic AMPA receptors mediated by enhanced recycling of internalized AMPA receptors back to the postsynaptic membrane. Here we report a novel, physiologically relevant NMDA receptor-independent mechanism that drives increased AMPA receptor recycling and LTP. This pathway requires the metabotropic action of kainate receptors and activation of G-protein, protein kinase C and phospholipase C. Like classical LTP, kainate receptor-dependent LTP recruits recycling endosomes to spines, enhances synaptic recycling of AMPA receptors to increase their surface expression and elicits structural changes in spines, including increased growth and maturation. These data reveal a new and previously unsuspected role for postsynaptic kainate receptors in the induction of functional and structural plasticity in the hippocampus.

\section{Introduction}

The precise dynamic regulation of the number, composition and distribution of postsynaptic AMPA receptors (AMPARs) is essential for synaptic transmission and plasticity. Classical LTP, which has been characterised extensively in many brain areas, requires the activation of NMDA receptors (NMDARs) ${ }^{1}$. An equivalent NMDAR-dependent LTP can be induced in cultured hippocampal neurons by brief exposure to the NMDAR co-agonist glycine, which elicits the insertion of AMPARs into the postsynaptic membrane and increases miniature excitatory postsynaptic currents (mEPSCs) ${ }^{2}$.

LTP involves both recycling-dependent increases in AMPAR surface expression at the postsynaptic membrane and increases in dendritic spine size ${ }^{3}$. Furthermore, stimuli that induce LTP in dispersed hippocampal neuronal cultures promote recycling and recruitment of transferrin receptor-positive recycling endosomes into spines. This repositioning of the endosomal-recycling compartment is critical for activity-dependent changes in spine morphology and provides a mechanistic link between structural and functional plasticity ${ }^{3,4}$.

Kainate receptors (KARs) are tetrameric assemblies of combinations of GluK1 - GluK5 subunits. They are present at both pre- and postsynaptic membranes where they perform distinct roles in modulating synaptic transmission, neuronal excitability and network activity ${ }^{5}$ ${ }^{6}$, and are implicated in processes ranging from neuronal development and differentiation to neurodegeneration and neuronal cell death ${ }^{5,7}$. In addition to direct ionotropic signalling, KARs also signal through the activation of $G$ proteins leading to PKC activation ${ }^{5,6,8-10}$. Under physiological glutamate release conditions, postsynaptic KAR metabotropic signalling in CA1 and CA3 pyramidal neurones inhibits the hyperpolarisation caused by the post-spike potassium current I (SAHP) ${ }^{11-14}$. 
We, and others, have shown previously that transient kainate (KA) stimulation modulates surface expression of KARs ${ }^{15-18}$ via changes in Rab11-dependent recycling within spines ${ }^{19}$. Here, we report that KA induces an NMDAR-independent increase in the synaptic surface expression of functional AMPARs, as well as the structural plasticity via a pathway that requires metabotropic signalling of postsynaptic GluK2-containing KARs and enhanced endosomal vesicle recycling in spines.

\section{Results}

\section{KAR activation increases surface expression of functional synaptic AMPARs.}

We first investigated the effects of KA application on AMPAR surface expression in the presence of tetrodotoxin (TTX, $0.5 \mu \mathrm{M})$, GYKI53655 (40 $\mu \mathrm{M})$ and L689560 (5 $\mu \mathrm{M})$ to suppress activity-dependent glutamate release and prevent activation of AMPARs and NMDARs respectively. Agonist stimulation of KARs (10 $\mu \mathrm{M} \mathrm{KA}, 3 \mathrm{~min})$ significantly increased surface expression of both GluA1 and GluA2 AMPAR subunits (Fig. 1A, p=0.001 and $p=0.004$, respectively), without altering the total number of AMPARs (Supplementary Fig.1A$B, p>0.09)$. This KAR-induced increase in plasma membrane expressed AMPARs was blocked by the competitive AMPAR/KAR antagonist CNQX (10 $\mu \mathrm{M}$; Fig. 1A, p>0.9).

To define if the KAR-induced increase in AMPAR surface expression occurred at spines, we immuno-labelled surface expressed GluA1 and GluA2 in non-permeabilised neurons (Fig. 1B) and calculated the spine/adjacent dendrite ratio. This was increased for both GluA1 and GluA2 following KAR stimulation ( $p=0.001$ for GluA1 and $p=0.003$ for GluA2), indicating preferential AMPAR surface expression in spines. Consistent with this, KAR-stimulation increased surface GluA1 and GluA2 colocalisation with the postsynaptic marker PSD95 (Fig. $1 \mathrm{C}, \mathrm{p}<0.001)$.

The increase in synaptic GluA1 and GluA2 was induced by a brief ( $3 \mathrm{~min}$ ) exposure of the cultured hippocampal neurons to all KA concentrations tested, ranging from $500 \mathrm{nM}$ to $20 \mu \mathrm{M}$ (Supplementary Fig. 1C-D, $p<0.001$ and $p<0.001$ respectively). We next tested if nanomolar concentrations of KA increased postsynaptic AMPAR-mediated mEPSCs in CA1 pyramidal neurons in hippocampal slices. KA concentrations below $3 \mu \mathrm{M}$ do not activate AMPARs in CA1 ${ }^{20}$, but, to fully exclude direct KA activation of AMPARs, we co-applied the AMPAR specific antagonist GYKI53655 (40 $\mu \mathrm{M})$ during the transient KA application. GYKI53655 was then washed out to obtain AMPAR mEPSCs. Co-application of GYKI53655 with $500 \mathrm{nM} \mathrm{KA}$ did not change mEPSC frequency (Supplementary Fig. 2A, $p>0.3$ ) but caused a marked increase in the amplitude of mEPSCs (Fig. 1D and Supplementary Fig. $3 A, p<0.004$. These data demonstrate a KAR-induced increase of functional postsynaptic AMPARs.

\section{KAR-mediated NMDAR-independent LTP.}


101 Increased postsynaptic AMPARs surface expression underpins LTP, so we investigated the 102 effects of KAR activation on synaptically evoked AMPAR-mediated excitatory postsynaptic 103 responses in rat hippocampal slices. KA (10 $\mu \mathrm{M}, 3 \mathrm{~min})$ caused a transient depression 104 followed by a progressive increase in AMPAR-mediated CA1 EPSCs (Supplementary Fig. $1054 \mathrm{~A} ; 169.8 \% \pm 31.4 \%, \mathrm{p}=0.01$ ) with no change in glutamate release probability determined by 106 the paired-pulse ratio (PPR; Supplementary Fig. 4B) in the continuous presence of $100 \mu \mathrm{M}$ 107 APV (to block NMDARs). At this concentration, however, KA activates AMPARs and could 108 affect their dynamics. Thus, we performed the experiment as above, but using $40 \mu \mathrm{M}$ 109 GYKI53655 (present prior to and during KA application to block AMPARs). Although in the 110 presence of GYKI53655 there was a significant difference in amplitude between the control 111 and KA conditions (KA, 100.8\% \pm 15.5\%; Control 54.6\% $\pm 8.0 \%, p=0.009$; Supplementary 112 Fig. 4C, unchanged PPRs are shown in Supplementary Fig. 4D and representative 113 responses shown in Supplementary Fig. 4E), GYKI53655 masked the time course of 114 AMPAR-mediated EPSCs. We therefore reduced the KA concentration to $500 \mathrm{nM}$, thereby 115 avoiding the need to block AMPARs with GYKI53655. Under these conditions, $500 \mathrm{nM} \mathrm{KA}$ 116 caused a progressive and persistent increase in AMPAR-mediated CA1 EPSCs (Figure 2A; $117133.1 \% \pm 11.4 \%, \mathrm{p}=0.02)$, reaching a peak approximately 10-15 min after the agonist 118 application. Again, we found no change in PPR (Supplementary Fig. 4F, p>0.3). Combined 119 with our mEPSC data, these evoked EPSC results indicate that KAR activation elicits 120 NMDAR-independent KA-induced LTP via increased surface expression of postsynaptic 121 AMPARs in CA1 hippocampal neurons.

122 Consistent with this, KA did not induce any potentiation in AMPAR-mediated field potentials 123 in the CA1 region of hippocampal slices from GluK2-knockout (GluK2 ${ }^{-1}$ ) mice $(95.7 \pm 3.6 \%$, $124 \mathrm{p}>0.05$, Supplementary Fig 5A). To exclude any possible developmental effects in the 125 GluK2-knockout mice, we tested the effect of acutely blocking KARs in wild-type mice using 126 the AMPAR/KAR blocker CNQX $(10 \mu \mathrm{M})$ prior to and during KA challenge, followed by 127 CNQX washout (Supplementary Fig. 5B). Compared to sham controls (CNQX only), the 128 recovery profile was unchanged (80.2 $\pm 3.4 \%$ vs. $78.7 \pm 9.5 \%$ for KA and control, $p>0.05)$.

129 We also confirmed that KA application potentiated AMPAR responses in hippocampal slices 130 obtained from adult, 3-month old mice $(124.7 \pm 9.6 \%, p<0.01)$ (Supplementary Fig. 5C-D), 131 indicating that KA-induced LTP is not developmentally restricted.

132 Using wild-type and GluK2-knockout mice, we next examined the KAR dependency of a 133 previously reported NMDAR-independent form of LTP evoked by high frequency stimulation 134 (HFS) protocol, based on the modified procedure from ${ }^{21,22}$ (illustrated in schematic form in 135 Fig. 2B and described in Methods). As shown in Supplementary Fig. 6A-C, field potential 136 recordings revealed robust LTP in acute hippocampal slices from wild-type mice (185.8 \pm 
$26.1 \%$ vs.106.1 $\pm 5.2 \%$ in test vs. control pathway, $\mathrm{p}=0.007$ ), whereas a reduced LTP was obtained in GluK2-knockout mice (170.3 $\pm 17.3 \%$ vs.106.2 $\pm 5.7 \%$ in test vs. control pathway, $p=0.03$ ). To isolate the NMDAR-independent component of this LTP, we used 50 $\mu \mathrm{M}$ D-APV, which completely blocked LTP in the GluK2-knockout $(106.3 \pm 5.0 \%$ vs. $99.4 \pm$ $4.6 \%$ in test vs. control pathway, $p>0.5)$, but not in WT mice (128.5 $\pm 11.4 \%$ vs.107.7 $\pm 5.0 \%$ in test vs. control pathway, $p<0.02$ ). No changes in PPR were observed (Supplementary Fig. 6D). These data confirm that activation of GluK2-containing KARs by synaptically released glutamate induces NMDAR-independent and KAR-dependent hippocampal LTP.

Consistent with previous reports ${ }^{22}$, the NMDAR-independent component of this HFS LTP in wild-type mice was blocked by the L-type calcium blocker nifedipine (110.45 $\pm 3.66 \%$ vs. $104.05 \pm 3.81 \%, p>0.05$, test vs. control pathway, Supplementary Fig. 7A). As in previous experiments, no change in the PPR was detected (Supplementary Fig. 7B).

Although widely used, HFS does not correspond to intrinsic in vivo patterns of hippocampal activity. Therefore, to determine if KAR-dependent LTP can be induced by more physiological stimuli, we used a protocol based on hippocampal sharp-wave/ripple-like stimulation pattern (RL-LTP; illustrated in schematic form in Fig. 2C) ${ }^{23}$. In wild-type mice, the RL-LTP protocol resulted in a progressive potentiation that peaked 10-15 min after stimulation (Fig. 2D left panel; $166.2 \pm 8.5 \%$ vs $101.1 \pm 2.9 \%$ in test vs control pathway, p<0.001). In GluK2-knockout mice (Fig. 2D right panel; representative traces in Fig. 2E), there was a significantly reduced LTP $(139.7 \pm 7.5 \%$ vs.100.6 $\pm 2.7 \%$ in test vs. control pathway, $p<0.001$, and $p=0.03$ compared to wild-type mice, Fig. 2F). Importantly, however, while RL-LTP (although at reduced level) was still detected in wild-type mice in the presence of $50 \mu \mathrm{M}$ D-APV (Fig 2D left panel; $120.9 \pm 2.3 \%$ vs $99.5 \pm 2.8 \%$ in test vs control pathway, $\mathrm{p}<0.001), \mathrm{RL}-\mathrm{LTP}$ was completely prevented in GluK2-knockout mice $(99.1 \pm 4.0 \%$ vs $99.2 \pm$ $3.8 \%$, test vs control pathway, $p=0.98$, and $p=0.002$ compared to wild-type mice).

162 D-APV is a competitive antagonist, therefore, to exclude the theoretical possibility that the 163 intense RL-LTP stimulation paradigm could lead to glutamate accumulation in synaptic cleft 164 that could out-compete D-APV, thereby allowing NMDAR-dependent LTP, we used a 165 previously described strategy ${ }^{24}$. We first blocked the NMDARs with MK-801 (20 $\left.\mu \mathrm{M}\right)$, a use166 dependent blocker (in nominal $0 \mathrm{mM} \mathrm{Mg}^{2+}$ aCSF, to facilitate the NMDAR activation). Then, 167 once the full blockade was achieved, we reintroduced ordinary aCSF, continuously 168 supplemented with MK-801 $(20 \mu \mathrm{M})$ and D-AP5 $(50 \mu \mathrm{M})$. Robust potentiation still occurred in 169 wild-type mice, further confirming the KAR-dependent component of RL-LTP (142.6 \pm 0.6 vs. $17098.2 \pm 9.3 \%$ in test vs. control pathway, p<0.01; Supplementary Fig. 7C-D). 
171 Finally, to further discount any possible confounding developmental issues in GluK2-

172 knockout mice, we performed the RL-LTP experiment in wild-type mice while acutely

173 blocking KARs using CNQX (10 $\mu \mathrm{M}$, present prior to and during LTP induction, followed by

174 washout), similarly to the approach used in Supplementary Fig. 5B. No RL-LTP was induced

175 after full inhibition by CNQX, i.e. both test and control pathways gradually recovered with

176 similar temporal profiles (Supplementary Fig. 7E, minutes 37-41: $20.9 \pm 4.6 \%$ vs. $22.3 \pm 3.2$,

$177 p>0.05$; minutes 57-61: $75.1 \pm 6.00 \%$ vs. $70.9 \pm 6.46 \%, p>0.05$; minutes 77-81: $100.4 \pm$

$1780.7 \%$ vs. $100.5 \pm 2.6 \%, p>0.05$, test vs. control pathway). These results provide

179 compelling evidence that physiologically relevant stimulation of GluK2-containing KARs can

180 induce NMDAR-independent LTP in the hippocampus.

181 Next, using rat hippocampal slices, we performed within-slice comparisons of test and control

182 pathway responses to RL-LTP and KA application. First we induced RL-LTP (normalized

183 fEPSP slope in test and control pathway $=123.1 \pm 1.8 \%$ and $98.1 \pm 1.6 \%$, respectively,

$184 \mathrm{p}<0.001$, Supplementary Fig. 8A-C). We then subjected the slice to bath application of KA

185 (500 nM, $3 \mathrm{~min}$ ), which caused a robust increase in control pathway to levels equivalent to

186 the RL-LTP in the test pathway (normalized fEPSP slope in test and control pathway = 132.9

$187 \pm 2.4 \%$ and $131.7 \pm 3.6 \%$, respectively; $\mathrm{p}=0.78$ ). Importantly, however, there was no further

188 increase in the pathway previously subjected to RL-LTP. These data also demonstrate that

189 the extent of agonist-induced LTP in field recordings is comparable to that achieved by 190 patch-clamp recording.

191 Our data from GluK2 ${ }^{-1-}$ mice indicate that LTP is not saturated in the presence of D-APV 192 because RL-LTP induced significantly stronger LTP in WT mice. In a complementary 193 approach, we directly tested if agonist and electrical stimulation-induced KAR-LTP share a 194 common mechanism using a previously established occlusion protocol ${ }^{25}$.

195 We initially induced RL-LTP in one pathway (black circles, Supplementary Fig. 8D, $100.9 \pm$ $1962.05 \%$ vs. $133.4 \pm 10.00 \%, p<0.05)$ and then bath applied KA (500 nM, $3 \mathrm{~min})$. As expected, 197 and consistent with the data in Supplementary Fig. 8A, the pathway that had not been 198 subjected to RL-LTP was potentiated by kainate (white circles, Supplementary Fig. 8D-E, $199118.1 \pm 4.0 \%, p<0.01)$. Importantly, KA did not cause additional potentiation in the pathway 200 previously exposed to RL-LTP (black circles, $128.0 \pm 11.2 \%, p>0.05$ ). The stimulation 201 intensity of the test pathway (white circles) was then adjusted to normalise it to its basal level 202 (101.6 $\pm 5.7 \%, p>0.05)$ and the RL-LTP protocol was then delivered to this pathway 203 (Supplementary Fig. 8D-E). Since no further potentiation was observed (97.6 $\pm 9.3 \%, p>$ 204 0.05), this inverse occlusion experiment confirms that KA-induced potentiation and RL-LTP 205 share a common mechanism. 
NMDAR-dependent LTP elicits structural changes in spine shape and increased spine size ${ }^{4}$. Correspondingly, transient KA application robustly increases spine density and maturity (Fig. $3 A-C)$, More specifically, there are increases in mature spine size $(65.1 \% \pm 14 \%, p<0.001$; Fig. $3 A$ ) and numbers of dendritic protrusions (68.9\% $\pm 25 \%, p<0.001$; Fig. 3B), as well as enhanced transition from stubby to mushroom spines (18.7\% $\pm 4 \%, p<0.001$; Fig. $3 C)$.

NMDAR-dependent LTP also enhances generalised endosomal recycling of cargo proteins and membrane within the spine ${ }^{3,4}$. To investigate if the same mechanisms underlie KARdependent LTP, we monitored transferrin-Alexa594 (Tf-A594) labelled recycling endosomes. Tf-A594 endosomes distribute mainly at the base of spines in non-stimulated control neurons. Following transient KA application, however, Tf-A594 endosomes translocate from the dendritic shaft to the spine head (Fig. 4A). Furthermore, expression of a dominant negative version of the recycling endosome-associated small GTPase Rab11 (Rab11dn), which blocks NMDAR-dependent LTP ${ }^{26}$, prevented the KAR-evoked recruitment of recycling endosomes into spines (Fig. 4B) and blocked the increases in the head diameter of mushrooms spines ( $p<0.0001$ for KA - control, and Rab11wt - Rab11dn; Fig. 4C).

To confirm the role of recycling in KAR-dependent LTP, we used surface biotinylation assays in combination with primaquine to selectively inhibit recycling ${ }^{27}$ or monensin to block both recycling and lysosomal degradation ${ }^{28}$. As expected, both drugs prevented the KAR-induced increase in GluA1 and GluA2 surface expression (Supplementary Fig. 9A-B, p<0.001). We next quantified surface expression of GluA1 and GluA2 in spines and adjacent shaft regions. Inhibiting recycling with primaquine, monensine or Rab11dn prevented the KAR-induced change in the spine:dendrite ratio of AMPAR surface expression (Supplementary Fig. 10A-C, $p=0.18$ for GluA1 and $p=0.24$ for GluA2, $p=0.80$ GluA1 and $p=0.34$ GluA2, and $p=0.03$ for GluA1 and $p=0.02$ for GluA2). These data indicate that both NMDAR- and KAR-dependent LTP require the recruitment and enhanced recycling of endosomal vesicles in spines.

KAR-dependent LTP is mediated via a non-canonical G-protein-associated signalling pathway.

Although some mechanistic details are still lacking, it is now clear that KARs signal via Gprotein-dependent pathways to increase intracellular calcium $\left[\mathrm{Ca}^{2+}\right]_{\mathrm{i}}$, and activate protein kinase $C(P K C)$ and phospholipase $C(P L C)^{5,6,9,10,29}$. Since increased $\left[\mathrm{Ca}^{2+}\right]_{i}$ is required for LTP, ${ }^{30,31}$ we tested the source of $\left[\mathrm{Ca}^{2+}\right]_{i}$ increase in KAR-dependent LTP using the extracellular chelator EDTA and the membrane permeant chelator BAPTA-AM. The presence of BAPTA-AM, but not EDTA, during the KA application blocked the KAR-evoked 
241 Supplementary Fig. 11A; BAPTA-AM; GluA1, p=0.5; GluA2 p= 0.10: EDTA; GluA1 p=0.009;

242 GluA2 $p<0.001$ ) and the associated changes in the structural plasticity (Fig. $5 \mathrm{C}$ and

243 Supplementary Fig. 11B, EDTA; $p<0.001$; BAPTA-AM $p=0.61$ ). Similarly, preincubation with

244 PKC inhibitor chelerythrine or the PLC inhibitor U73122 blocked the KAR-induced increase in 245 GluA1 and GluA2 expression at synapses (Fig. 5A, B; controls without KA shown in 246 Supplementary Fig. 11A-B; U73122: GluA1 p=0.56; GluA2 $p=0.32$; chelerythrine: GluA1 $247 p=0.54$, GluA2 p= 0.78), the increase in the spine size (Fig. 5C and Supplementary Fig. 11B, 248 U73122: $p=0.42$; chelerythrine: $p=0.48$ ), as well as the agonist-evoked KAR-dependent LTP 249 in electrophysiological recordings (100.5 $\pm 1.4 \%, p>0.05$ for U73122 and $100.7 \pm 5.8 \%$, 250 p $>0.05$ for chelerythrine, Fig. 5D, E).

251 We also analysed PKC and PLC activity in cells after triggering KAR-dependent LTP. Brief 252 KAR stimulation (3 min, 500nM) elicited a nearly 2-fold increase in PLC and PKC activity 253 compared to unstimulated cells (Fig. 5F). Furthermore, the extent of PKC activation in KAR254 dependent LTP is similar to stimulation by the phorbol ester PMA $(0.5 \mu \mathrm{M}, 3 \min , \mathrm{p}=0.4)$. The 255 KAR-induced activation of PKC and PLC was prevented by CNQX and BAPTA-AM, but not 256 by the presence of EDTA during KA application (Fig. 5F: PKC: +CNQX: p=0.037, +EDTA: $257 p=0.81,+B A P T A-A M, p=0.002,+$ Chelerythrine $p=0.038$. For PLC: +CNQX: $p=0.047,+E D T A:$ $258 \mathrm{p}=0.84,+$ BAPTA-AM: $p=0.056,+\mathrm{U} 73122 \mathrm{p}=0.006)$. Finally, in addition to EGTA (5 mM) 259 application only during KA application, we continuously applied (30 min during and after KA 260 application) the selective $\mathrm{Ca}^{2+}$ chelator EGTA to bind extracellular $\mathrm{Ca}^{2+}$ or nifedipine to block 261 L-type voltage-gated $\mathrm{Ca}^{2+}$ channels (VGCCs). Continuous application of either drug 262 prevented the KA-induced increase in GluA1 and GluA2 surface expression at synapses 263 (Supplementary Fig. 11C, D; $p<0.001$ ), suggesting a delayed role for extracellular $\mathrm{Ca}^{2+}$ in 264 KAR-LTP. Importantly, inhibiting Group I /II metabotropic glutamate receptors with the 265 specific antagonist MCPG did not affect KAR-dependent increases in synaptic AMPARs or 266 spine size. (Supplementary Fig. 12A, $B ; p<0.001$ and $p=0.018$ ). These data demonstrate that 267 KAR-LTP requires activation of PKC and PLC and intracellular calcium release, consistent 268 with a KAR-mediated metabotropic signalling pathway.

269 Furthermore, we performed the same experiments as those shown in Figure 1C, but with the 270 preincubation in the presence of G-protein inhibitor pertussis toxin (PTX) $(1 \mu \mathrm{g} / \mathrm{mL}, 1$ hour). 271 PTX prevented KA-induced activation of both PLC and PKC (Fig. 6A, p=0.03 and p=0.012 272 respectively). PTX also blocked the increased colocalization of GluA1 and GluA2 with PSD95 273 following the KA challenge (Fig. 6B, GluA1, p=0.93; GluA2 p=0.47, compare with Fig. 1C). 274 Furthermore, incubation of hippocampal slices with PTX prior to recording mEPSCs 275 prevented the KAR-induced increase in AMPAR mEPSC amplitude in CA1 pyramidal 276 neurons (Fig. 6C, p=0.34, compare with Fig. 1D), with no change in the frequency 
277 (Supplementary Fig. 13A p >0.1). Correspondingly, agonist-evoked and electrically stimulated

278 KAR-dependent LTP (Fig. 6D-E and Supplementary Fig. 13B-D, p=0.69 and p=0.3, compare

279 with Fig. 2A), as well as structural plasticity (Fig. 6F and Supplementary Fig. 13E, p=0.4,

280 compare with Fig. 3A) were prevented by preincubation with PTX, again indicating the

281 requirement for a metabotropic action for KARs.

282 Both our imaging and functional data using nifedipine suggest that following initial LTP 283 induction that requires intracellular $\mathrm{Ca}^{2+}$, extracellular calcium entry through L-type VGCCs 284 plays a role in maintaining KAR-LTP. Moreover, VGCC currents can be modulated by G285 protein activation ${ }^{32}$. We therefore used patch-clamp electrophysiology to measure VGCC $286 \mathrm{Ca}^{2+}$ currents. KA application (500 nM, $3 \mathrm{~min}$ ) increased VGCC currents in control conditions $287(127.4 \pm 16.1 \%, n=5)$, but there was no increase in slices that had been preincubated with 288 PTX $(90.4 \pm 4.5 \%, n=4, p<0.05$, Supplementary Fig. 14).

289 Substitution of extracellular $\mathrm{Na}^{+}$with an equimolar concentration of the non-permeant cation 290 N-methyl-D-glucamine (NMDG) prevents KAR channel conductance, but does not impede 291 metabotropic KAR activity ${ }^{9}$. Replacing $\mathrm{Na}^{+}$with NMDG does not block the KAR-induced 292 increase in GluA1 and GluA2 colocalization with PSD95 and spine size (Supplementary 293 Fig.15A-B; GluA1, $p=0.004$, GluA2, $p<0.001$ and $p=0.009$ for spine size), further confirming 294 that ionotropic activity is not required for KAR-dependent LTP. The KAR antagonist UBP310 295 has been reported to inhibit KAR ionotropic activity, but not KAR-metabotropic signalling, via 296 a mechanism that likely involves an action beyond simple competitive antagonism ${ }^{33}$. This 297 effect is unlikely to be due to different subunit compositions since most KARs in the brain 298 comprise GluK2/5 combinations. We anticipate that future studies will uncover the 299 mechanisms underlying this selective inhibition of ionotropic over metabotropic KAR activity. 300 Nonetheless, consistent with the documented selectively ionotropic action,UBP310 (10 $\mu \mathrm{M})$ 301 did not block KA-induced increases in PLC and PKC activity (Fig. 7A, $p=0.74$ and $p=0.94$ 302 compared to KA) nor did it prevent KAR-dependent LTP (Fig. 7B-C and Supplementary 303 Fig.15C-D $p=0.01$ and $p=0.006)$ and structural spine plasticity (Fig.7D, $p=0.03)$ ).

304 Taken together, this array of complementary and mutually supportive data provide 305 compelling evidence that KAR channel activity is not required for KAR-dependent LTP, but is 306 instead underpinned by KAR-mediated metabotropic signalling.

\section{Discussion}

308 Here we report that KAR activation can elicit a previously unanticipated form of NMDAR309 independent LTP. This occurs via a metabotropic KAR pathway that recruits endosomal 310 recycling machinery from the dendritic shaft into the spine to alter post-endocytic GluA1 and 311 GluA2 sorting and exocytosis back to the spine plasma membrane. 
313 We have shown previously that transient KA application can increase KAR surface 314 expression ${ }^{16}$ and enhance spine growth by altering post-endocytic sorting and enhanced

315

316

317

318

319

320

321

322

323

324 recycling mechanisms ${ }^{19}$. Furthermore, KARs regulate neurite outgrowth ${ }^{16,34,35}$, as well as filopodia and nascent spinule development ${ }^{36}$. Here we show that transient KAR activation augments recycling and surface expression of AMPARs, increases AMPAR colocalisation with PSD95 in spines and increases the amplitude of AMPAR mEPSCs. Consistent with postsynaptic mechanisms, the probability of neurotransmitter release was unchanged. Furthermore, using two different stimulation protocols, we demonstrate that synaptic activation of GluK2-containing KARs underlies the increases in the evoked AMPAR-mediated responses. These results reveal a novel and physiologically relevant form of postsynaptic KAR-dependent, NMDAR-independent LTP.

\section{KAR activation increases synaptic recycling and spine size}

In parallel with increased AMPAR-mediated neurotransmission, NMDAR-dependent LTP elicits the formation and enlargement of dendritic spines to consolidate neural circuitry ${ }^{37,38}$. Recycling endosomes are recruited to deliver membrane material directly within spines for structural plasticity ${ }^{3,4}$, providing a mechanistic link for coupling changes in spine size to the regulation of AMPAR-mediated transmission and LTP ${ }^{39}$. Like NMDAR-dependent LTP, KARdependent LTP requires translocation of Rab11-positive recycling endosomes from the dendritic shaft into spines. Moreover, overexpression of dominant negative Rab11, which blocks NMDAR-dependent LTP ${ }^{40}$, prevents the KAR-evoked redistribution of recycling endosomes to spines and blocks KAR-dependent LTP. This involvement of Rab11 in NMDAR-dependent and KAR-dependent LTP indicates shared mechanisms in both pathways.

\section{Metabotropic actions of KARs mediate KAR-dependent LTP}

Metabotropic KAR signalling was first identified through the KAR-mediated modulation of GABA release, which does not require KAR channel activation, but is prevented by inhibition of G-protein and PKC activity ${ }^{8}$. Subsequently, KAR-dependent inhibition of the slow afterhyperpolarizing potential (SAHP), which enhances neuronal excitability, was also shown to be mediated by metabotropic KAR signalling ${ }^{11}$. Although there is now a wealth of experimental support for metabotropic action of both pre- and postsynaptic KARs (for reviews see ${ }^{5,6,41}$ ), many questions remain. For example, the identity of the KAR subunit conferring metabotropic action is unclear because the literature is contradictory and no KAR subunits contain conventional G-protein binding motifs. Nonetheless, it is now generally accepted that metabotropic KAR signalling is PTX sensitive and thus involves Go rather than 
$347 \mathrm{Gq}$ protein activation. Accordingly, a recent report has suggested that the KAR subunit

348 GluK1 can associate directly with a Go protein $\alpha$ subunit and that this association is 349 responsible for the metabotropic effects of KARs ${ }^{10}$. Our results now reveal an entirely novel 350 role for metabotropic KAR signalling in regulating AMPAR trafficking, spine morphology and 351 NMDAR-independent LTP.

\section{KAR-dependent LTP}

353 LTP at CA1 hippocampal synapses is not uniform and comprises a range of NMDAR354 dependent and -independent plasticity mechanisms ${ }^{42}$. Given the crucial importance of 355 plastic changes in the brain, this array of pathways provides a dynamic, flexible and reliable 356 system to ensure the continuity of neuronal network and brain function. Our identification of a 357 novel postsynaptic KAR-dependent LTP adds to these important system traits. Ripple-like 358 high-frequency patterns of activity ( $\sim 200 \mathrm{~Hz}$ for $\sim 100 \mathrm{~ms}$, repeating at $\sim 1 \mathrm{~Hz})$ occur in 359 immobile awake animals and during slow wave sleep ${ }^{43}$. These patterns generally occur in 360 conjunction with large-amplitude sharp waves and ripple-related activity in vivo is implicated 361 in LTP that underlies memory consolidation in the hippocampus ${ }^{44,45}$. Here, we show for the 362 first time that this strong and physiologically relevant ripple-like activity LTP induction 363 protocol (RL-LTP) is mediated via GluK2-containing KARs.

364 It is notable that the induction of KAR-LTP and the previously reported agonist-evoked 365 increase in surface expression of GluK2-containing KARs share a similar time course that 366 reaches a plateau $10-15 \mathrm{~min}$ after stimulation ${ }^{16}$. This profile correlates with the delayed 367 NMDAR-independent component of HFS-induced LTP ${ }^{21}$ and shares dependency on VGCC 368 activation. Moreover, G-protein potentiation of VGCC activity is sensitive to PTX and requires 369 PKC activation and increases in $\left[\mathrm{Ca}^{2+}\right]_{\mathrm{i}}{ }^{32}$, consistent with KAR-metabotropic actions 370 modulating VGCC activity in NMDAR-independent LTP. These features are similar to the role 371 of mGluR5 receptor metabotropic signalling which, by facilitating L-type VGCC activity via 372 intracellular $\mathrm{Ca}^{2+}$ release, contributes to NMDAR-independent forms of LTP ${ }^{46}$. It is important 373 to note that the rise in $\left[\mathrm{Ca}^{2+}\right]_{\text {i }}$, presumably mediated via IP3 receptors, can facilitate VGCC 374 activity and that VGCC activity and the influx of extracellular calcium can prolong the 375 temporal profile and frequency of intracellular $\mathrm{Ca}^{2+}$-release events ${ }^{47}$. This reciprocal 376 feedback system fits with our imaging and electrophysiological experiments with nifedipine 377 and can extend beyond the kainate stimulation. We anticipate that future work will explore 378 this feedback system in more detail by combining simultaneous multiphoton imaging and 379 electrophysiology in brain slices.

\section{Concluding remarks}


381 Here we describe an entirely new pathway in which direct activation of postsynaptic KARs

382 induces LTP. These data show that KAR metabotropic signalling facilitates information

383 transfer and synaptic integration by two parallel mechanisms, namely the short-term

384 regulation of excitability ${ }^{13,14}$ and long term increase in synaptic efficacy via LTP. Both

385 mechanisms are induced by high frequency stimulation of KARs and require PKC. Given that

386 KARs are highly expressed during the neuronal circuit formation, and that their dysfunction is

387 implicated in many neurological diseases including epilepsy ${ }^{48}$ and intellectual disability ${ }^{49}$,

388 we anticipate that our findings will have far reaching implications.

\section{Acknowledgements}

390 We are grateful for financial support from the ERC (Proposal $n^{\circ}$ 232881), MRC 391 (MR/L003791), BHF (PG/14/60/31014) and BBSRC (BB/K014366 and BB/K014358) to JMH;

392 EMBO Fellowships to MIGG (ALTF 224-2009 and ASTF 438-2011) and MMP (ASTF 232-

393 2011); MRC (MR/M023729/1) to MMP; the Centre National de la Recherche Scientifique, the

394 Conseil Régional d'Aquitaine, the Labex BRAIN and the Fundacao para a Ciencia e a 395 Tecnologia to CM and SVS; the Czech Science Foundation (GACR): 17-02300S; 396 P304/12/G069) and Research Project of the AS CR RVO (67985823) to LV; the Department 397 of Science and Technology (DST) - Young Scientist Scheme (SERB/LS-779/2013) to JPC. 398 We are grateful to P. Rubin and N. Grosjean for excellent technical support, A. Singh for his 399 help in some follow-up experiments and to J. Esteban (CBMSO, Madrid) for providing Rab 400 constructs.

\section{Author contributions} MIGG designed and performed the biochemistry and imaging experiments and participated 403 in electrophysiological experiments; MMP designed and performed agonist and stimulation 404 evoked electrophysiology and participated in imaging experiments. SVS did 405 electrophysiology in wild-type and GluK2 ${ }^{-1-}$ mice hippocampal slices; CM provided knockout 406 mice and extensive advice; JPC performed the MK-801/D-APV and CNQX dual pathway 407 electrophysiological experiments. LV provided facilities and reagents and helped analyse the 408 electrophysiological data. JMH instigated the study and provided overall supervision and 409 management. JMH, MIGG and MMP designed the study, analysed the data and wrote the 410 paper. All authors discussed the results and commented on the manuscript.

\section{Author Information}

412 The authors declare no competing financial interests. 


\section{References}

415 1. Malenka, R.C. \& Bear, M.F. LTP and LTD: an embarrassment of riches. Neuron 44, 541621 (2004).

417 2. Lu, W., et al. Activation of synaptic NMDA receptors induces membrane insertion of 418 new AMPA receptors and LTP in cultured hippocampal neurons. Neuron 29, 243-254. (2001).

3. Park, M., Penick, E.C., Edwards, J.G., Kauer, J.A. \& Ehlers, M.D. Recycling endosomes supply AMPA receptors for LTP. Science 305, 1972-1975 (2004).

422

4. Park, M., et al. Plasticity-induced growth of dendritic spines by exocytic trafficking from

5. Contractor, A., Mulle, C. \& Swanson, G.T. Kainate receptors coming of age: milestones

6. Lerma, J. \& Marques, J.M. Kainate receptors in health and disease. Neuron 80, 292311 (2013).

7. González-González, I.M., et al. Kainate Receptor Trafficking. WIRES Membrane Trasnsport and Signalling 1, 31-44 (2012).

8. Rodriguez-Moreno, A. \& Lerma, J. Kainate receptor modulation of GABA release involves a metabotropic function. Neuron 20, 1211-1218. (1998).

9. Rozas, J.L., Paternain, A.V. \& Lerma, J. Noncanonical signaling by ionotropic kainate receptors. Neuron 39, 543-553 (2003).

10. Rutkowska-Wlodarczyk, I., et al. A Proteomic Analysis Reveals the Interaction of GluK1 Ionotropic Kainate Receptor Subunits with Go Proteins. J Neurosci 35, 51715179 (2015).

11. Melyan, Z., Wheal, H.V. \& Lancaster, B. Metabotropic-mediated kainate receptor regulation of IsAHP and excitability in pyramidal cells. Neuron 34, 107-114 (2002).

12. Fisahn, A., Heinemann, S. \& McBain, C.J. The Kainate Receptor Subunit GluR6 Mediates Metabotropic Regulation of the Slow and Medium AHP Currents in Mouse Hippocampal Neurons. J Physiol (2004).

13. Melyan, Z., Lancaster, B. \& Wheal, H.V. Metabotropic regulation of intrinsic excitability by synaptic activation of kainate receptors. J Neurosci 24, 4530-4534 (2004).

14. Ruiz, A., Sachidhanandam, S., Utvik, J.K., Coussen, F. \& Mulle, C. Distinct subunits in heteromeric kainate receptors mediate ionotropic and metabotropic function at hippocampal mossy fiber synapses. J Neurosci 25, 11710-11718 (2005).

15. Rivera, R., Rozas, J.L. \& Lerma, J. PKC-dependent autoregulation of membrane kainate receptors. EMBO J 26, 4359-4367 (2007). 
16. Martin, S., Bouschet, T., Jenkins, E.L., Nishimune, A. \& Henley, J.M. Bidirectional regulation of kainate receptor surface expression in hippocampal neurons. J Biol Chem 283, 36435-36440 (2008).

17. Selak, S., et al. A role for SNAP25 in internalization of kainate receptors and synaptic plasticity. Neuron 63, 357-371 (2009).

18. Carta, M., et al. CaMKII-dependent phosphorylation of GluK5 mediates plasticity of kainate receptors. EMBO J 32, 496-510 (2013).

19. Gonzalez-Gonzalez, I.M. \& Henley, J.M. Postsynaptic Kainate Receptor Recycling and Surface Expression Are Regulated by Metabotropic Autoreceptor Signalling. Traffic (2013).

20. Bureau, I., Bischoff, S., Heinemann, S.F. \& Mulle, C. Kainate receptor-mediated responses in the CA1 field of wild-type and GluR6-deficient mice. J Neurosci 19, 653663 (1999).

21. Grover, L.M. \& Teyler, T.J. Normal-Methyl-D-Aspartate Receptor-Independent LongTerm Potentiation in Area CA1 of Rat Hippocampus - Input-Specific Induction and Preclusion in a Non-Tetanized Pathway. Neuroscience 49, 7-11 (1992).

22. Grover, L.M. \& Teyler, T.J. Two components of long-term potentiation induced by different patterns of afferent activation. Nature 347, 477-479 (1990).

23. Behrens, C.J., van den Boom, L.P., de Hoz, L., Friedman, A. \& Heinemann, U. Induction of sharp wave-ripple complexes in vitro and reorganization of hippocampal networks. Nat Neurosci 8, 1560-1567 (2005).

24. Grover, L.M. Evidence for postsynaptic induction and expression of NMDA receptor independent LTP. J Neurophysiol 79, 1167-1182 (1998).

25. Huang, Y.Y. \& Malenka, R.C. Examination of TEA-induced synaptic enhancement in area CA1 of the hippocampus: the role of voltage-dependent $\mathrm{Ca} 2+$ channels in the induction of LTP. J Neurosci 13, 568-576 (1993).

26. Brown, T.C., Correia, S.S., Petrok, C.N. \& Esteban, J.A. Functional compartmentalization of endosomal trafficking for the synaptic delivery of AMPA receptors during long-term potentiation. J Neurosci 27, 13311-13315 (2007).

27. van Weert, A.W., Geuze, H.J., Groothuis, B. \& Stoorvogel, W. Primaquine interferes with membrane recycling from endosomes to the plasma membrane through a direct interaction with endosomes which does not involve neutralisation of endosomal pH nor osmotic swelling of endosomes. European journal of cell biology 79, 394-399 (2000).

28. Mollenhauer, H.H., James Morré, D. \& Rowe, L.D. Alteration of intracellular traffic by monensin; mechanism, specificity and relationship to toxicity. Biochimica et Biophysica Acta (BBA) - Reviews on Biomembranes 1031, 225-246 (1990). 
29. Sihra, T.S., Flores, G. \& Rodriguez-Moreno, A. Kainate Receptors: Multiple Roles in Neuronal Plasticity. Neuroscientist (2013).

30. Lynch, G., Larson, J., Kelso, S., Barrionuevo, G. \& Schottler, F. Intracellular injections of EGTA block induction of hippocampal long-term potentiation. Nature 305, 719-721 (1983).

31. Malenka, R.C., Kauer, J.A., Zucker, R.S. \& Nicoll, R.A. Postsynaptic calcium is sufficient for potentiation of hippocampal synaptic transmission. Science 242, 81-84 (1988).

32. Zong, X. \& Lux, H.D. Augmentation of calcium channel currents in response to $G$ protein activation by GTP gamma $S$ in chick sensory neurons. J Neurosci 14, 48474853 (1994).

33. Pinheiro, P.S., et al. Selective block of postsynaptic kainate receptors reveals their function at hippocampal mossy fiber synapses. Cereb Cortex 23, 323-331 (2013).

34. Marques, J.M., et al. CRMP2 tethers kainate receptor activity to cytoskeleton dynamics during neuronal maturation. J Neurosci 33, 18298-18310 (2013).

35. Lanore, F., et al. Deficits in morphofunctional maturation of hippocampal mossy fiber synapses in a mouse model of intellectual disability. J Neurosci 32, 17882-17893 (2012).

36. Tashiro, A., Dunaevsky, A., Blazeski, R., Mason, C.A. \& Yuste, R. Bidirectional regulation of hippocampal mossy fiber filopodial motility by kainate receptors. A twostep model of synaptogenesis. Neuron 38, 773-784 (2003).

37. Engert, F. \& Bonhoeffer, T. Dendritic spine changes associated with hippocampal longterm synaptic plasticity. Nature 399, 66-70 (1999).

38. Matsuzaki, M., et al. Dendritic spine geometry is critical for AMPA receptor expression in hippocampal CA1 pyramidal neurons. Nat Neurosci 4, 1086-1092 (2001).

39. Matsuzaki, M. Factors critical for the plasticity of dendritic spines and memory storage. Neurosci Res 57, 1-9 (2007).

40. Wang, Z., et al. Myosin Vb mobilizes recycling endosomes and AMPA receptors for postsynaptic plasticity. Cell 135, 535-548 (2008).

41. Lerma, J. Kainate receptor physiology. Current opinion in pharmacology 6, 89-97 (2006).

42. Malenka, R.C. \& Nicoll, R.A. Long-term potentiation--a decade of progress? Science 285, 1870-1874 (1999).

43. Ylinen, A., et al. Sharp wave-associated high-frequency oscillation $(200 \mathrm{~Hz})$ in the intact hippocampus: network and intracellular mechanisms. J Neurosci 15, 30-46 (1995). 
521

522

523

524

525

526

527

528

529

530

531

532

533

534

535

536

537

538

539

540

541

542

543

544

545

546

547

548

\section{9}

546

47

44. O'Neill, J., Senior, T. \& Csicsvari, J. Place-selective firing of CA1 pyramidal cells during sharp wave/ripple network patterns in exploratory behavior. Neuron 49, 143-155 (2006).

45. Ego-Stengel, V. \& Wilson, M.A. Disruption of ripple-associated hippocampal activity during rest impairs spatial learning in the rat. Hippocampus 20, 1-10 (2010).

46. Kato, H.K., Kassai, H., Watabe, A.M., Aiba, A. \& Manabe, T. Functional coupling of the metabotropic glutamate receptor, InsP3 receptor and L-type Ca2+ channel in mouse CA1 pyramidal cells. J Physiol 590, 3019-3034 (2012).

47. Miyazaki, K. \& Ross, W.N. Ca2+ sparks and puffs are generated and interact in rat hippocampal CA1 pyramidal neuron dendrites. J Neurosci 33, 17777-17788 (2013).

48. Crepel, V. \& Mulle, C. Physiopathology of kainate receptors in epilepsy. Current opinion in pharmacology 20, 83-88 (2015).

49. Motazacker, M.M., et al. A defect in the ionotropic glutamate receptor 6 gene (GRIK2) is associated with autosomal recessive mental retardation. American journal of human genetics 81, 792-798 (2007).

50. Petrovic, M.M., et al. Inhibition of post-synaptic Kv7/KCNQ/M channels facilitates longterm potentiation in the hippocampus. PLoS One 7, e30402 (2012).

40

41

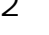

44 
551 Fig 1. KA increases AMPAR surface expression.

552 A, Immunoblots show the KA-evoked increased surface expression of GluA1 and GluA2,

553 which was blocked by CNQX. Data were quantified as $\%$ of control, $n=3$ independent 554 experiments. Dot-plots on the right indicated values for the individual experiments. Unless 555 otherwise indicated, all imaging and biochemistry experiments are performed in the

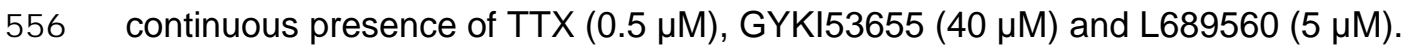

557 B, Confocal images showing surface GluA1 and GluA2 in the dendritic shaft and spine (scale 558 bar $1 \mu \mathrm{m})$ and cumulative frequency plots of spine/dendrite ratios. $\mathrm{n}=11-16$ cells per 559 condition, 3 independent experiments.

560 C, Co-localization of GluA1 or GluA2 (red) and PSD95 (green). Scale bar $1 \mu \mathrm{m}$. Graphs 561 Show Pearson's coefficients for the co-localization. The black line in the whisker plot boxes 562 indicates the median. $\mathrm{n}=10-15$ cells per condition, 3 independent experiments.

563 D, mEPSCs from CA1 pyramidal cells in hippocampal slices in the continuous presence of $564 \mathrm{APV}$ and $\pm \mathrm{GYKI}$ and $\pm \mathrm{KA}$. Quantification of the data using cumulative distribution plots of 565 mEPSC amplitudes and whisker plots. $n=4$ cells for control, $n=5$ cells for $K A$ from $n=4$ 566 animals.

567 In all experiments shown in B, C and D, data acquisition and analysis were performed in 568 blind with respect to the treatment.

\section{Fig 2. KAR activation induces LTP.}

571 A, Effects of KA on normalized evoked EPSC amplitudes and sample traces from CA1 572 neurons in the presence of D-APV in mice hippocampal slices. $n=5$ cells from 5 animals for 573 control and $n=6$ cells from 6 animals for kainate.

574 B-C, Schematic representations of the HFS and the RL-LTP induction protocols.

575 D, Normalized fEPSP slope in WT and GluK2 ${ }^{--}$mice subjected to RL-LTP. The symbols for 576 experiments without APV are squares (black for control and white for test pathway), whereas 577 for experiments with APV the symbols are circles (black for control and white for test 578 pathway). Arrow indicates point of RL-LTP stimulation. WT: $n=8$ slices from 8 animals; WT + 579 AP5: $n=9$ slices from 3 animals; GluK2 ${ }^{-1}: n=8$ slices from 8 animals; GluK2-/- + AP5 n= 8 580 slices from 8 animals.

581 E, Representative traces for D. 
F, Normalized fEPSP slope values 21-30 min post LTP protocol.

583 In all experiments shown in B, C and D, data acquisition and analysis were performed in 584 blind with respect to the treatment or genotype of the animal.

585

Fig 3. KAR-LTP induces structural plasticity.

587 A, KAR-induced increase in spine size. Right panel shows quantification of spine area after $588 \mathrm{KA}(\mathrm{A}) /$ area before $\mathrm{KA}\left(\mathrm{A}_{0}\right)$ versus time. The period of $\mathrm{KA}$ application is indicated by the 589 black bar. $n=4-6$ cells per condition, 3 independent experiments, Scale bars $1 \mu \mathrm{m}$.

590 B-C, Time-lapse experiments showing KA-induced increase in the number of protrusions and 591 enhanced transition from stubby to mushroom spines. The number of protrusions was 592 quantified before KA application $\left(\mathrm{N}_{0}\right)$ and at the indicated times $(\mathrm{N})$ in $10 \mathrm{~mm}$ segments of 593 dendrites \pm KA. The period of KA application is indicated by the black bar. $n=3-6$ cells per 594 condition, 3 independent experiments. Scale bars $4 \mu \mathrm{m}$.

595 In all experiments, data analysis was performed in blind with respect to the treatment.

596

597 Fig 4. KAR-LTP recruits rab11-recycling endosomes to spines.

598 A, KA (red arrow) recruits transferrin-Alexa594 (red) labelled recycling endosomes to spines. 599 GFP was expressed to visualise morphology. Time is indicated in seconds. Right panel 600 shows the quantification of the proportion of endosomes in head or shaft, $n=5-6$ cells per 601 condition, 3 independent experiments. Scale bar $1 \mu \mathrm{m}$.

602 B, Dominant negative Rab11 prevents recruitment of recycling endosomes into spines. Tf603 A488 positive endosomes (green) in neurons expressing RFP-rab11wt or dn (red). n=6-8 604 cells per condition, 3 independent experiments. Scale bar $1 \mu \mathrm{m}$.

$605 \mathrm{C}$, Images of spines before $(\mathrm{t}=0)$ and $30 \mathrm{~min}(\mathrm{t}=30)$ after $\mathrm{KA}+/$ - CNQX in neurons expressing 606 GFP, Rab11wt or Rab11dn. Frequency distribution plots of individual spine diameters before 607 (black, $\mathrm{t}=0$ ) and after (grey dotted line, $\mathrm{t}=30 \mathrm{~min}$ ) KA. $\mathrm{n}=4-5$ cells per condition, 3 608 independent experiments. Scale bar $1 \mu \mathrm{m}$.

609 In all experiments, data analysis was performed in blind with respect to the treatment.

610

611 Fig 5. KAR-LTP requires intracellular calcium increase, PKC and PLC activation.

612 A-B, BAPTA-AM, U73122 and chelerythrine, but not EDTA, block KAR-mediated increase in 613 co-localization of surface GluA1 or GluA2 (red) and PSD95 (green). Scale bar $1 \mu \mathrm{m}$. Box- 
614 and-whisker plots show range of Pearson's coefficient of controls (see also Supplementary 615 Fig. 5) and KA treated cells (KA). Black line in the boxes indicates the median. $n=6-15$ cells 616 per condition, 3 independent experiments. Data analysis was performed in blind with respect 617 to the pharmacological treatment.

$618 \mathrm{C}$, Images of spines before $(\mathrm{t}=0)$ and $30 \mathrm{~min}(\mathrm{t}=30)$ after indicated drugs $+/-\mathrm{KA}$. 619 Corresponding graphs on the right show frequency distribution of individual spine diameters 620 before (black, $t=0$ ) and after (grey dotted line, $t=30$ min) KA. $n=4$ cells per condition, 3 621 independent experiments. Note that extracellular chelation of calcium by EDTA does not 622 prevent KAR-mediated increase in structural plasticity. Data analysis was performed in blind 623 with respect to the pharmacological treatment.

624 D-E, Effects of KA on normalized evoked EPSC amplitudes and sample traces from CA1 625 neurons in the presence of chelerythrine (upper panel) or the PLC inhibitor U-73122 (bottom 626 panel) in mice hippocampal slices. $n=6$ slices from 2 animals for chelerythrine and $n=7$ slices 627 from 2 animals for U-73122.

628 F, Box-and-whisker plots show range of fold increase in PLC (right) and PKC (left) activity 629 after KA challenge. PLC or PKC activity was normalized to controls in the presence of the 630 indicated drugs. Black line in the boxes indicates the median. $n=4-6$ independent 631 experiments.

632

633 Fig 6. KAR-LTP requires KAR metabotropic signalling.

634 A, Box-and-whisker plots show range of fold increase in PLC (right) and PKC (left) in cells 635 preincubated with PTX. PLC or PKC activity was normalized to control and performed in 636 parallel with the experiment in figure 5C. Black line in the boxes indicates the median. $n=4-6$ 637 independent experiments.

638 B, Co-localization of surface GluA1 or GluA2 (red) and PSD95 (green). Scale bar 1 um. Box639 and-whisker plots of Pearson's coefficients of colocalization indicate that the metabotropic 640 pathway inhibitor PTX blocks KAR-evoked increase in surface AMPARs. Black line in the 641 boxes indicates the median. $n=9-13$ cells per condition, 3 independent experiments.

642 C, PTX blocks KAR-mediated increase in CA1 mEPSC amplitude (compare to Fig. 1D). $n=3$ 643 cells from 3 animals. Examples of traces pretreated with PTX before and after GYKI53655 644 (control) and shown before and after KA plus GYKI53655 (KA+GYKI). Graphs show 645 cumulative frequency distribution of mEPSC amplitudes and box-and-whisker plots in insets 646 indicating range. 
647 D, Preincubation with PTX impaired the KA-induced increase of normalized evoked EPSC

648 amplitudes in WT mice hippocampal slices (compare with Fig. 2A). Sample traces are shown 649 before and after $K A$ challenge. $n=6$ cells from 3 animals per condition.

650 E, Normalized fEPSP slope recorded in WT-mice hippocampal slices pretreated with PTX.

651 Arrow indicates point of LTP induction. $n=7$ slices from 4 animals.

$652 \mathrm{~F}$, Images of spines before $(\mathrm{t}=0)$ and $30 \mathrm{~min}(\mathrm{t}=30)$ after $\mathrm{KA}$ in cells treated with PTX. 653 Frequency distribution of individual spine diameters before (black, $t=0$ ) and after ( $r e d, t=30$

654 min) KA. $n=3$ cells per condition, 3 independent experiments.

655 In all experiments data analysis was performed in blind with respect to the treatment.

656

657 Fig 7. KAR-LTP does not require ionotropic KAR activation.

658 A, Box-and-whisker plots show fold increase in PLC (right) and PKC activity (left) in the 659 presence of the ionotropic KAR inhibitor UBP310 $(10 \mu \mathrm{M})$. PLC or PKC activity was 660 normalized to control and performed in parallel with the experiments in figure $5 \mathrm{C}$. Black line 661 in the boxes indicates the median. $n=6$ independent experiments.

662 B, UBP310 $(10 \mu \mathrm{M})$ did not impair the KA-induced increase of normalized evoked EPSC 663 amplitudes in WT mice hippocampal slices (compare with Fig. 2A). Sample traces are shown 664 before and after KA challenge. $n=5$ cells from 4 animals.

665 C, Normalized fEPSP slope recorded in WT mice hippocampal slices in the presence of 666 UBP310 $(10 \mu \mathrm{M})$. Arrow indicates time point of LTP induction. $\mathrm{n}=11$ slices per condition from 6676 animals.

$668 \mathrm{D}$, Images of spines before $(\mathrm{t}=0)$ and $30 \mathrm{~min}(\mathrm{t}=30)$ after KA in cells treated UBP310 $(10 \mu \mathrm{M})$. 669 Frequency distribution of individual spine diameters before (black, $t=0$ ) and after ( $r e d, t=30$ $670 \mathrm{~min}) \mathrm{KA} . \mathrm{n}=4-6$ cells per condition, 3 independent experiments. Data analysis was performed 671 in blind with respect to the pharmacological treatment.

672 

A Control

$-3 \mathrm{~min} 0 \mathrm{~min} 12 \mathrm{~min} 18 \mathrm{~min} 36 \mathrm{~min}$

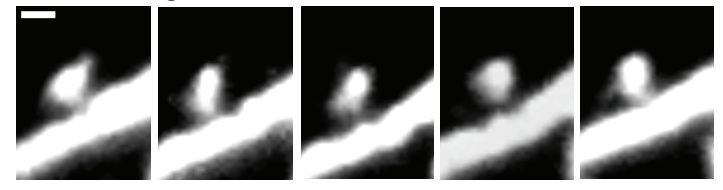

$\mathrm{KA} \downarrow$

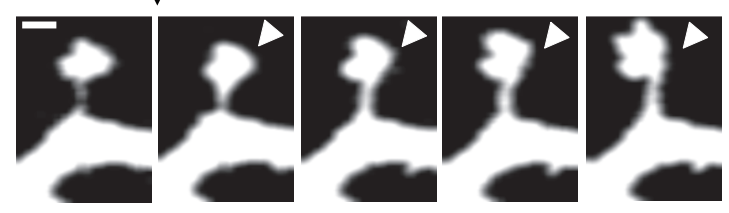

B Control

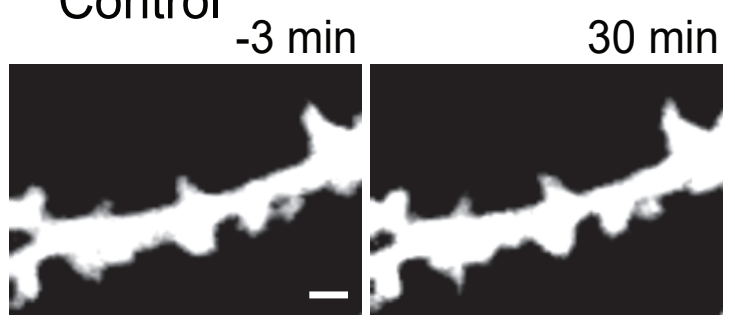

KA

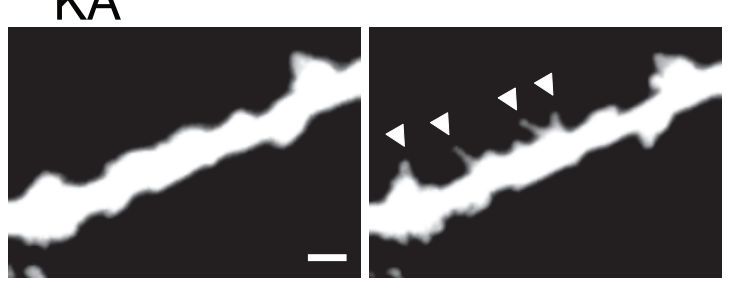

$$
\begin{aligned}
& \text { - Control } \\
& \left.\begin{array}{r}
1.8 \\
1.6 \\
1.4 \\
1.2 \\
1.0
\end{array}\right] \bullet \Phi^{\circ} \bullet \bullet
\end{aligned}
$$

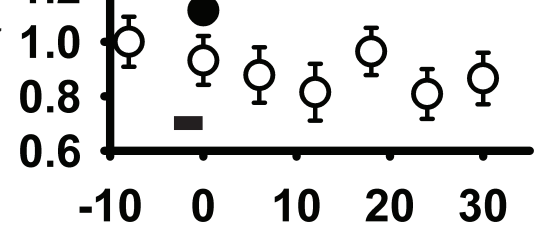

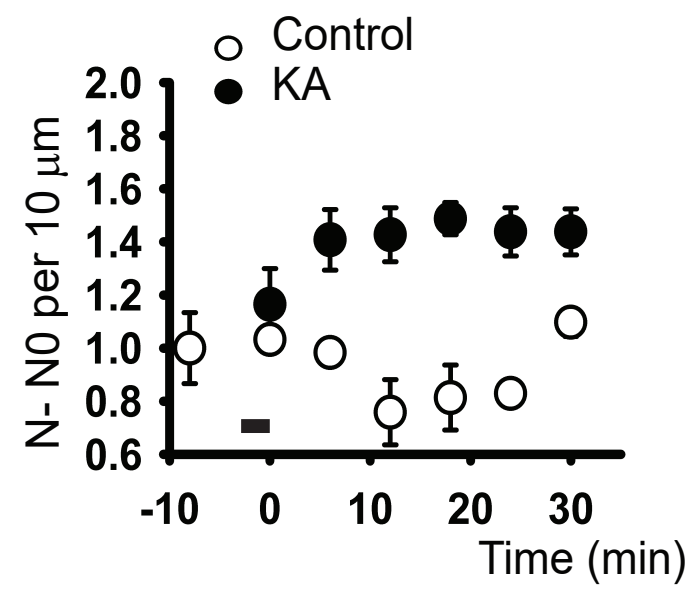

C
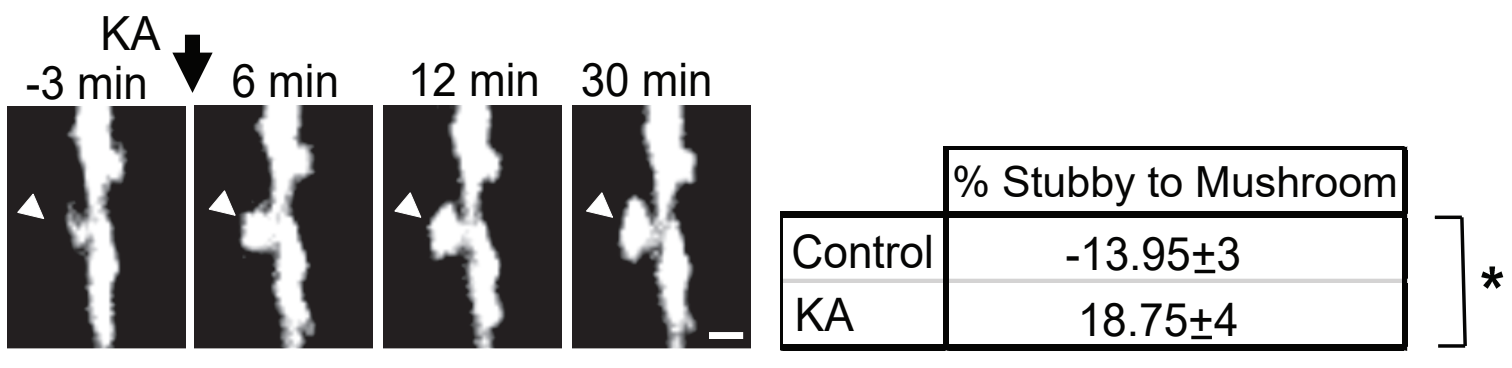


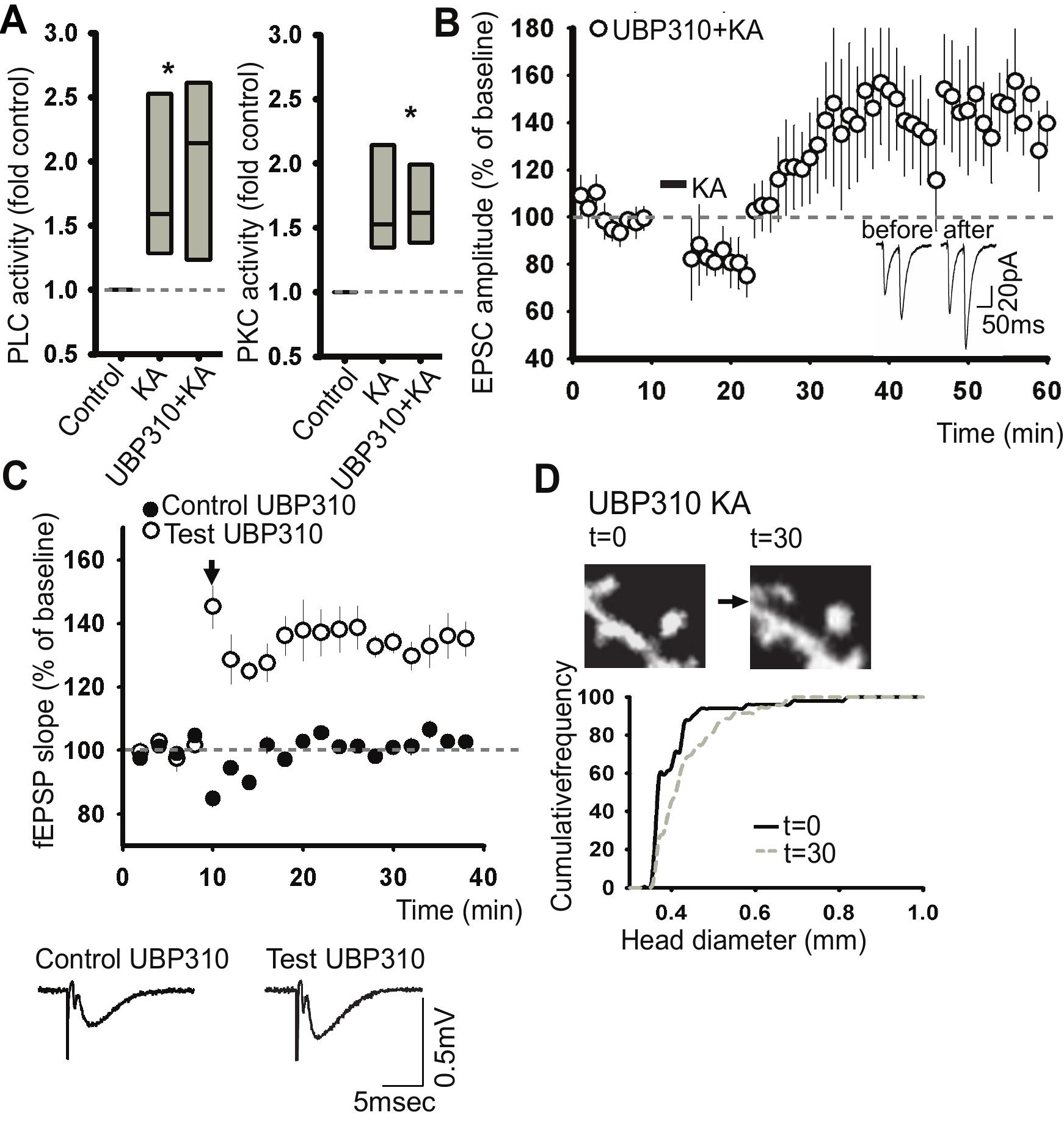


